\title{
Gain-Enhanced LTCC System-on-Package for Automotive UMRR Applications
}

\author{
F. A. Ghaffar, M. U. Khalid, A. Shamim, K. N. Salama \\ Electrical Engineering Program \\ King Abdullah University of Science and Technology (KAUST) \\ Thuwal, Saudi Arabia
}

\begin{abstract}
A novel LTCC based SoP for automotive radar applications is presented. For the first time a combination of a relatively low dielectric constant LTCC substrate and a high dielectric constant LTCC superstrate has been incorporated to enhance the overall gain of the module. The superstrate can provide additional protection to the integrated circuits (IC) in the harsh automotive environment. A custom cavity in the LTCC substrate can accommodate the IC, which feeds an aperture coupled patch antenna array. The cavity is embedded below the ground plane that acts as a shield for the IC from antenna radiation. It is estimated that with mere $10 \mathrm{dBm}$ of transmitted RF power the miniature $S o P$ module (sized $2.0 \mathrm{~cm} x$ $2.0 \mathrm{~cm} \times 0.22 \mathrm{~cm}$ ) can communicate up to $67 \mathrm{~m}$. The design's compactness, robustness, transmission power and resultant communication range are highly suitable for UMRR applications.
\end{abstract}

\section{INTRODUCTION}

Ever since the introduction of the first short range radar (SRR) system in the 2005 Mercedes S class model, interest in the automotive radar applications at the unlicensed $24 \mathrm{GHz}$ (Industrial, Scientific and Medical) band has been gathering a great deal of momentum with an increasing number of applications being envisaged [1], [2]. The Universal Medium Range Radar (UMRR) is one such example, which operates in the $24 \mathrm{GHz}$ band, and is generally utilized for advanced automotive driver assistance applications such as high precision parking aid, blind spot detection and high sensitivity pre-crash detection [3]. In addition, UMRR can be employed as a support for cut-in, stop and go situations in the high range Adaptive Cruise Control (ACC) systems [4]. However, the above mentioned radar applications are still limited to very expensive vehicles due to their high cost implementations [5].

A suitable low cost solution for such automotive radars is the System-on-Package ( $\mathrm{SoP}$ ) platform that can remove the barrier against a speedy introduction of such systems into the lower class, high-volume car market. SoP integrates multiple functions into a single, compact, low cost and high performance packaged module. It reduces the system size and cost immensely by transforming millimeter-scale discrete components into micrometer or nanometer-scaled embedded thin-film components. In the SoP domain, the Low Temperature Co-fired Ceramic (LTCC) technology offers many attractive features and possibilities such as the arbitrary number of layers, which not only allows embedded passives but also facilitates their vertical integration with other RF components [6]. Finally, the low loss nature of the LTCC substrates at microwave and millimeter-wave frequencies makes them very suitable for efficient antenna design [7], [8]. However, not much work has been done so far on the LTCC based automotive radars. In [9], an LTCC based automotive radar system at $24 \mathrm{GHz}$ has been demonstrated. However, it employs an eight-patch antenna array with a complex feed network, which renders the system to be bulky and inefficient. Moreover the design in [9] does not cater for harsh automotive environments.

Our previous work has demonstrated that the aperturecoupled technique is very suitable for LTCC medium [10]. We have also demonstrated that antenna gain can be enhanced manifolds utilizing a magnetic superstrate in LTCC SoP design [11]. This paper presents a $24 \mathrm{GHz}$ LTCC automotive radar SoP comprising of an integrated array of aperture coupled patch antennas and a thin high dielectric constant superstrate layer. For the first time, the gain resonance method [12] has been incorporated in LTCC, which is perfectly suited to the multilayer nature of this medium. This superstrate layer not only enhances the array gain but also adds to the robustness of the design by providing protection against severe conditions. Unlike [9], the design is simple, efficient and cost effective yet provides almost similar performance. The miniaturized module can easily fit into car bumpers or side mirrors etc. The paper highlights the design steps with some key results and also discusses some important design tradeoffs.

\section{CONCEPT}

The goal of this work is to integrate the RF circuits, embedded passives including antenna, and interconnects into a single compact package utilizing advanced LTCC technology. The concept of this SoP is shown in Figure 1. It employs a unique combination of two different dielectric constant LTCC 
layers. This combination allows the first practical implementation of the gain resonance method in LTCC medium. The substrate is realized in a relatively low dielectric constant $\left(\varepsilon_{\mathrm{r} 1}\right)$ LTCC material CT 707 and the superstrate makes use of a high dielectric constant $\left(\varepsilon_{\mathrm{r} 2}\right)$ LTCC material. The material properties of both types of LTCC are listed in Table 1. Each layer's fired thickness is $100 \mu \mathrm{m}$. The bottom substrate (SUBS1) is five layers thick with a four layer thick cavity to accommodate the Microwave and Millimeter-Wave Integrated circuit (MMIC). The MMIC is a flip-chip, which rests on the solder balls in the cavity. The output of the MMIC is connected to the feed microstrip line on a single layer thick middle substrate (SUBS2) through a solder ball and via combination. There is a two layer thick middle substrate (SUBS3) in between the feed microstrip and the ground plane. The ground plane contains an aperture, which is excited by the feed microstrip line. The top substrate (SUBS 4) is five layers thick and contains the patch antenna array. Finally, a four layers thick superstrate is placed above SUBS4 with an air gap of $0.4 \mathrm{~mm}$ between them to enhance the gain of the antenna array and improve the robustness of the module. The air gap is realized through four posts of CT 707 material.

\begin{tabular}{|c|c|c|}
\hline \multicolumn{2}{|c|}{ Table 1 - Properties of LTCC Materials } \\
\hline \multirow{2}{*}{ Properties } & \multicolumn{2}{|c|}{ LTCC Materials } \\
\cline { 2 - 3 } & CT707 & CT767 \\
\hline $\begin{array}{c}\text { Relative Dielectric } \\
\text { Constant }\end{array}$ & 6.39 & 68.7 \\
\hline Loss Tangent & 0.00481 & 0.00173 \\
\hline $\begin{array}{c}\text { Thickness of each } \\
\text { layer }(\mu \mathrm{m})\end{array}$ & 100 & 100 \\
\hline
\end{tabular}

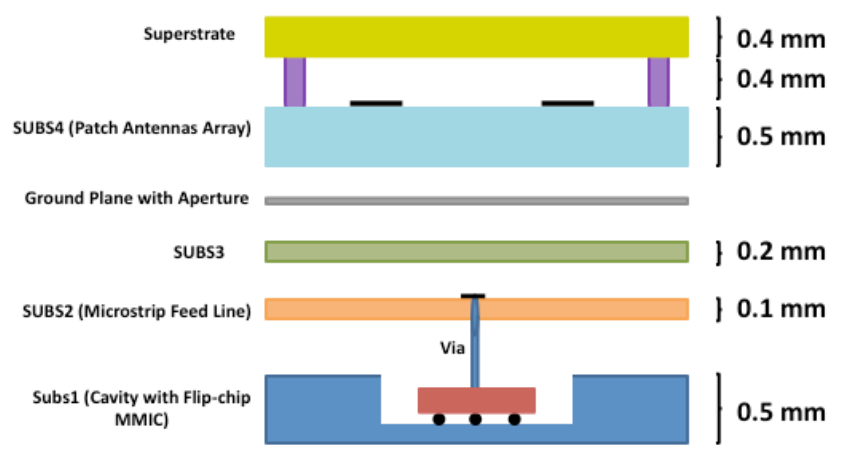

Figure $1-$ SoP concept

\section{ANTENNA ARRAy DESIGN}

The aperture coupled patch topology utilizes a common ground plane between the radiating antenna and the feed line. For this work, aperture coupling is employed mainly because the ground plane in between the patch antenna array and the MMIC acts as a shield for the circuits. A limitation of this technique is that the aperture in the ground plane can radiate considerably in the backward direction. However, by choosing the right slot length with respect to the patch size can minimize this unwanted radiation.
Array designs are employed in order to increase the gain achieved from a single antenna element. More the number of antenna elements in the array, higher is the overall gain. However, tradeoffs are the added complexity of the feed network, which enhances the substrate losses and the larger size of the module because of the additional antenna elements. In this work, the array comprises only two aperture coupled patch antennas fed by a single microstrip split into two lines. The complete SoP has been designed in an EM simulator $\mathrm{HFSS}^{\mathrm{TM}}$.

At first a single patch antenna element is designed for the required center frequency of $24 \mathrm{GHz}$. The antenna is fed through the aperture in the ground plane, which in turn is fed through a microstrip line. The microstrip feed line is excited through a lumped port in HFSS ${ }^{\mathrm{TM}}$. The width of the feed line is $1 \mathrm{~mm}$, which corresponds to a characteristics impedance of $50 \Omega$. The length of the slot plays a vital role in determining the resonant frequency of the antenna and also helps in optimizing the input impedance of the antenna design. On the other hand, the slot width is critical in controlling the backward radiation from the antenna. The slot length and width are optimized to be $2.3 \mathrm{~mm}$ and $0.1 \mathrm{~mm}$ respectively. This helps in achieving the desired radiation pattern with minimum backward radiation as shown in Figure 2 (a). The length of the patch antenna is optimized to be $2.13 \mathrm{~mm}$ while its width is $2.4 \mathrm{~mm}$. A good match with an $\mathrm{S} 11$ of $-16 \mathrm{~dB}$ and a gain of $4.6 \mathrm{~dB}$ is attained at $24 \mathrm{GHz}$.

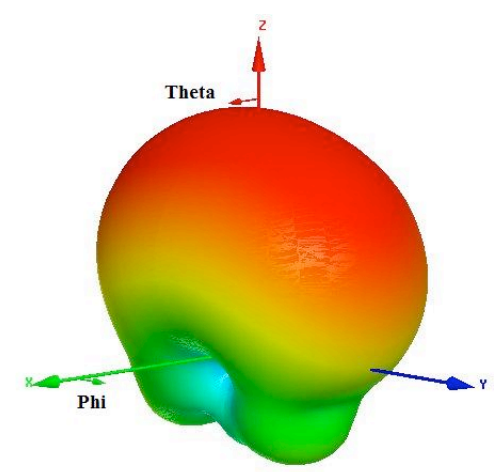

(a)

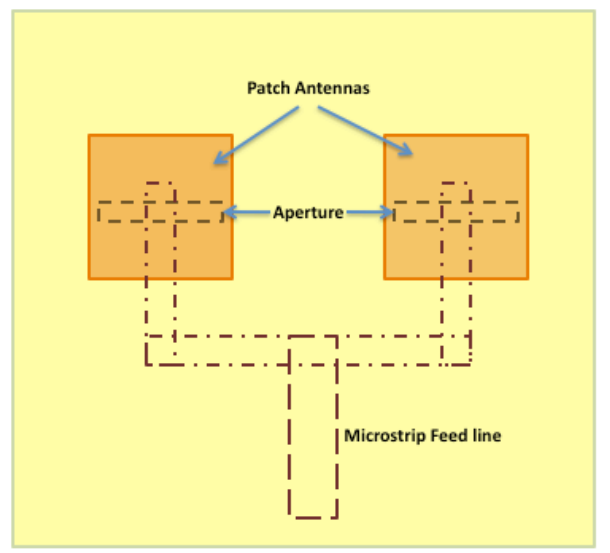

(b)

Figure 2 - Antenna Array (a) Design (b) Radiation Pattern of Single Element Patch 
As shown in Figure 2 (b), each element in the antenna array is fed through the a separate aperture in the ground plane, which in turn are excited through the microstrip lines divided in a T-fashion from the main microstrip feed line. The widths of these divided microstrip lines are $0.5 \mathrm{~mm}$ each and correspond to an impedance of $100 \Omega$. The two $100 \Omega$ lines connected in parallel match perfectly to the $50 \Omega$ main feed line. The antenna elements in the array have identical dimensions. The separation of $6.25 \mathrm{~mm}$ between the two patch elements corresponds to half free space wavelength $\left(0.5 \lambda_{\mathrm{o}}\right)$. The reflection coefficient of the single element and the complete array are shown in Figure 3. A good impedance match at $24 \mathrm{GHz}$ is observed in both the cases. The gain of the array is $5.8 \mathrm{~dB}$ as compared to $4.6 \mathrm{~dB}$ of the single element. The radiation pattern of the array, as shown in Figure 4, has narrowed a little from the broad bore-sight lobe in case of single element, which is expected due to increased gain. Moreover, the coupling between the two patch elements has resulted in enhanced back lobe levels. However, these can be reduced, if required, by further optimizing the aperture dimensions. Similarly, due to the thick LTCC substrate the gain of the two-patch LTCC array is slightly lower as the power is lost in surface waves. Higher gain can be achieved by replacing the thick substrate with thin LTCC layers having lower dielectric constant (close to air) as the antenna substrate.

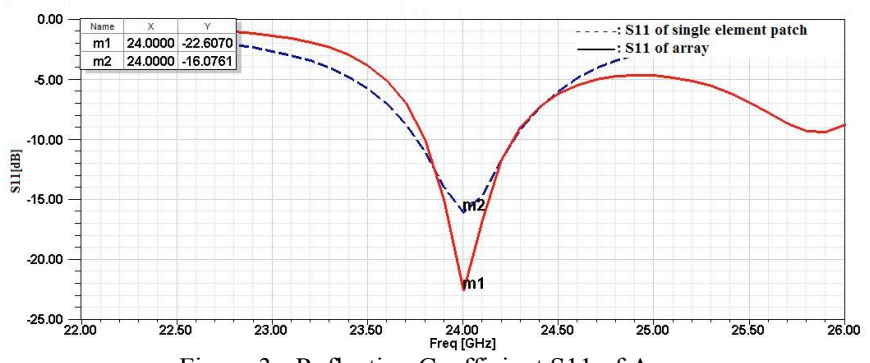

Figure 3 - Reflection Coefficient S11 of Array

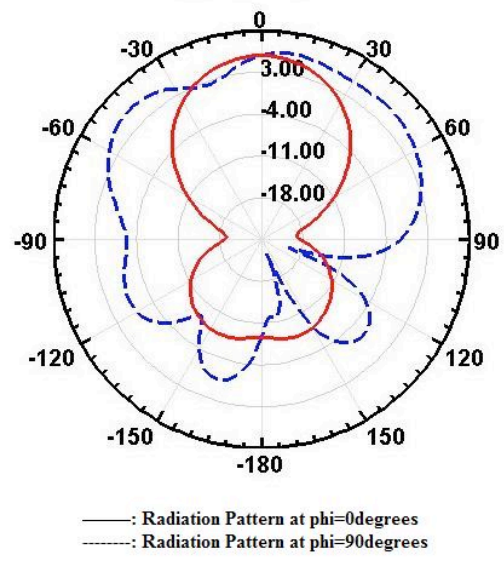

Figure 4 - Radiation Pattern of Aperture Coupled Array

\section{Gain EnHANCEMENT TeChNiQue}

The gain enhancement technique employed here is ideally suited to LTCC environment, as it requires the addition of a superstrate layer over the substrate. This is referred to as the resonance gain method and utilizes a superstrate with either relative permittivity, $\varepsilon_{\mathrm{r}}>1$ or relative permeability, $\mu_{\mathrm{r}}>1$. By choosing the layer thicknesses and antenna position properly, a very large gain may be realized at any desired angle. The gain varies proportionally to either $\varepsilon_{\mathrm{r}}$ or $\mu_{\mathrm{r}}$, depending on the configuration. However, the bandwidth is seen to vary inversely to gain so that a reasonable gain limit is actually established for practical antenna operation [11], [12]. The gain resonant condition for employing a $\varepsilon_{\mathrm{r}}>1$ superstrate is given as [12]:

$$
\begin{gathered}
\frac{\eta_{2} t}{\lambda_{o}}=\frac{2 p-1}{4} \\
\frac{\eta_{1} z_{o}}{\lambda_{o}}=\frac{2 n-1}{4} \\
\frac{\eta_{1} B}{\lambda_{o}}=\frac{m}{2}
\end{gathered}
$$

Where $\eta_{1}$ and $\eta_{2}$ are the refractive indices of the substrate and superstrate respectively. B and t represent the thicknesses of the substrate and superstrate respectively. Here $z_{0}$ is the height of the antenna with respect to the bottom of the substrate, $\mathrm{p}, \mathrm{m}$ and $\mathrm{n}$ are positive integers. For this design, the $\varepsilon_{\mathrm{r} 1}$ of 6.39 and the $\varepsilon_{\mathrm{r} 2}$ of 68.7 give $\eta_{1}$ of 2.52 and $\eta_{2}$ of 8.28 respectively. Choosing $p=1$ in (1) and calculating for 24 $\mathrm{GHz}$, the required $\mathrm{t}=0.377 \mathrm{~mm}$ or 4 layers. Similarly, selecting $\mathrm{n}$ and $\mathrm{m}$ equal to $1,(2)$ and (3) give $\mathrm{B}=2.47 \mathrm{~mm}$ or 25 layers, and $z_{0}=1.23 \mathrm{~mm}$ or 13 layers. Since 24 layers for substrate is not a cost effective solution, therefore the substrate thickness is restricted to the height required for locating the antenna, i.e. $1.3 \mathrm{~mm}$ or 13 layers. An air box is also included in between the substrate and superstrate like [11] and its thickness is estimated through parametric simulations in HFSS to determine the highest gain. The results are shown in Figure 5.

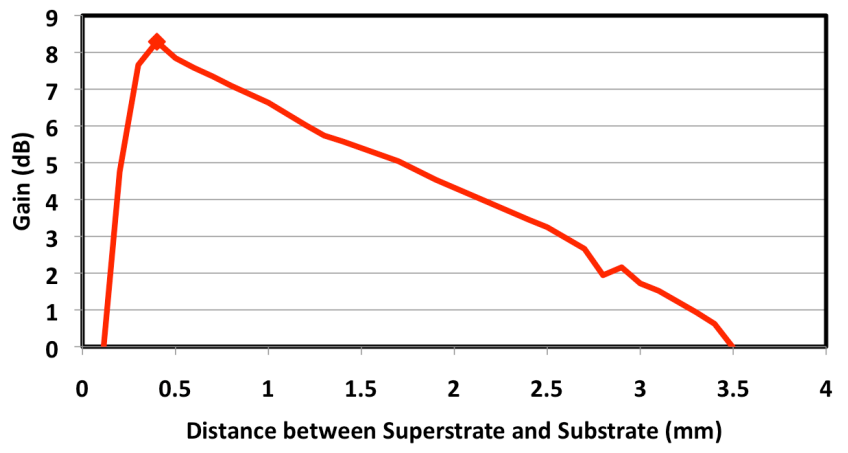

Figure 5- Gain vs. Distance between Superstrate and Substrate

As can be observed from Figure 5, the superstrate provides a gain enhancement of more than $2 \mathrm{~dB}$ at a height of $0.4 \mathrm{~mm}$ above the patch array. The final gain with the array and superstrate combination is $8.3 \mathrm{~dB}$. Besides the increase in the gain, the radiation pattern of the array is also affected by 
the superstrate loading and now has higher side lobe levels. This means that the spacing between the elements has to be re-adjusted after loading of the superstrate. Though the impedance of the antenna array is not much influenced by the superstrate loading, however a downward shift is observed in the $10 \mathrm{~dB}$ bandwidth, as shown in Figure 6. It is worth mentioning here that the gain of the array and superstrate combination can be increased manifolds, provided the substrate thickness is increased. However, the gain achieved with the present arrangement is enough for the medium range automotive radar applications. This can be further explained with the help of the following example.

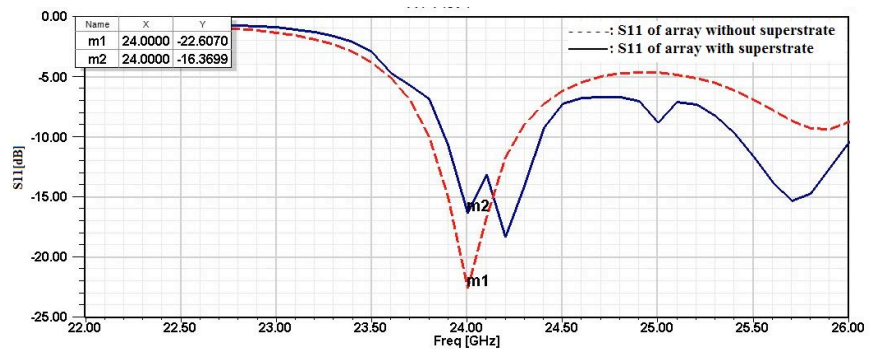

Figure 2 - Comparison of S1 1 of array with superstrate and without superstrate

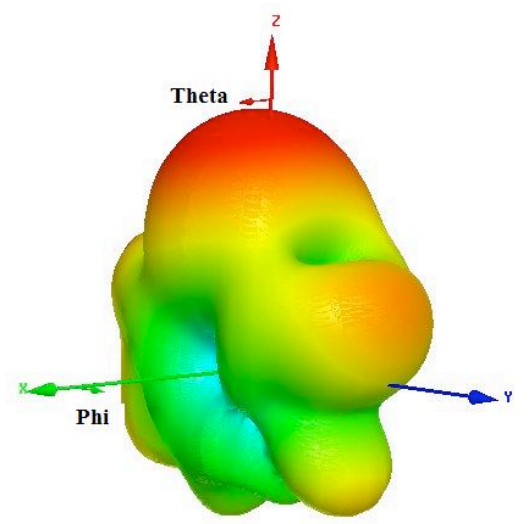

Figure 3 - Radiation Pattern of array with the superstrate

By employing the Friis transmission equation [13] the range of the SoP module can be determined. In a typical automotive radar application, the transmitting power $\left(\mathrm{P}_{\mathrm{t}}\right)$ from the transceiver is $10 \mathrm{dBm}$ at the antenna port [14]. The standard sensitivity of a typical receiver is around $-100 \mathrm{dBm}$. Keeping into account the fading effect margin of $30 \mathrm{~dB}$, let us assume the received power $\left(\mathrm{P}_{\mathrm{r}}\right)$ is $-70 \mathrm{dBm}$. Let us also consider that transmit and receive antennas are identical so the antenna gain for transmit $\left(G_{t}\right)$ and receive $\left(G_{r}\right)$ sides is $8.3 \mathrm{~dB}$. The resonant frequency is $24 \mathrm{GHz}$, which corresponds to a free space wavelength $\lambda_{0}$ of $12.5 \mathrm{~mm}$. By substituting all these values in Friis equation, the range can be calculated as follows.

$$
P_{r}=P_{t} \times G_{t} \times G_{r} \times\left(\frac{\lambda_{o}}{4 \pi R}\right)^{2}
$$

A communication range of $67 \mathrm{~m}$ is achieved, which is quite suitable for the medium range automotive radar applications. The range can be enhanced, if required, by further increase in antenna gain or transmitted power.

\section{CONCLUSION}

A unique design for an aperture coupled patch antenna array SoP realized in a mixed LTCC medium of low and high dielectric constants is presented. The substrate, realized in relatively low dielectric constant material, has a custom cavity to accommodate the MMIC. Shielding is provided by a ground plane between the circuits inside the cavity and the antennas on the top substrate layers. A superstrate in high dielectric medium not only enhances the gain of the module but also acts as an additional protection layer for harsh automotive environments. Analysis has revealed that a suitable communication range can be obtained through this design with standard transmission power levels for UMRR application.

\section{REFERENCES}

[1] R. Mende, H. Rohling, "New automotive applications for smart radar systems", GRS Bonn, September 2002.

[2] M. Kunert, R. Doerfler, W. Hosp, C. Preis, "24GHz UWB short range radars for vehicular applications", MWE-2003, November 2003.

[3] R. Mende, "The UMRR $24 \mathrm{GHz}$ radar sensor family for short and medium range applications" 2004.

[4] M. Klotz and H. Rohling, " $24 \mathrm{GHz}$ radar sensors for automotive applications", in Journal of Telecommunications and Information Technology, 2001.

[5] V. Cojocaru, H. Kurata, D. Humphrey, B. Clarke, T. Yokoyama, V. Napijalo, T. Young, T. Adachi, "A 24GHz Low-Cost, Long-Range, Narrow-Band, Monopulse Radar Front End System for Automotive ACC Applications", Microwave Symposium, 2007.

[6] Y.S. Bee, C. Z. Ning, A. C. W. Lu, V. Sunappan, L.L. Wai, “60-GHz LTCC Antenna Array with Microstrip to CPW Transition", Asia Pacific Microwave Conference, 2009

[7] S. Pinel et al., "System-on-package (SOP) architectures for compact and the low cost RF front-end modules," 33rd European Microwave Conference, 2003.

[8] J.S. Kim, S.H. Wi, J.C. Kim, J.G. Yook, "5 GHz Band LTCC Antenna for WMAN applications", Antennas and Propagation Society International Symposium, 2005.

[9] S. Holzwarth, R. Kulke, J. Kassner, "Integrated stacked patch antenna array on LTCC material operating at $24 \mathrm{GHz}$ [automotive radar applications]". Antennas and Propagation Society International Symposium, 2004

[10] A. Shamim, M. Arsalan, L. Roy, "Wireless interconnects between onchip and LTCC antennas for system in package applications", European Conference on Wireless Technology, 2008

[11] A. Shamim, G. Brzezina, M. Arsalan and L. Roy, "5.2 GHz differential LTCC antenna and balun for biomedical System in Package (SiP) application", in IWAT, 2007.

[12] D. Jackson, N. Alexopoulos, "Gain enhancement methods for printed circuit antennas," IEEE Transactions on APS, pp.976 - 987, Sept. 1985

[13] C. A. Balanis, Antenna Theory Analysis and Design, 2nd ed., John Wiley \& Sons, pp. 86, 1997.

[14] L. Eriksson et al., "A high performance automotive radar for automatic AICC," in Proc. 1995 Radar Conf., pp. 380-385. 